\title{
Novel luminescent dyes for confocal laser scanning microscopy used in Trematoda parasite diagnostics*
}

\author{
Elena Kirilova1 ${ }^{凶}$, Sanita Kecko², Ligita Mežaraupe², Inese Gavarāne1, Aleksandrs Pučkins¹, \\ llona Mickeviča', Ilze Rubeniną2, Sergejs Osipovs', Andrejs Bulanovs³, Mihails Pupinš̌2,4 and \\ Muza Kirjušina²
}

\begin{abstract}
1Daugavpils University, Institute of Life Sciences and Technology, Department of Biotechnology, Daugavpils, Latvia; ${ }^{2}$ Daugavpils University, Institute of Life Sciences and Technology, Department of Ecology, Daugavpils, Latvia; ${ }^{3}$ Daugavpils University, Institute of Life Sciences and Technology, Department of Technology, Daugavpils, Latvia; ${ }^{4}$ Latgales Zoo, Daugavpils, Latvia
\end{abstract}

\begin{abstract}
Benzanthrone derivates are now widely used in many industrial and scientific applications as dyes for polymers and textiles. In biochemical, biomedical and diagnostics investigations benzanthrone dyes are used as a lipophilic fluorescent probe since many benzanthrone derivates demonstrate bright fluorescence and they have ability to intercalate between membrane lipids. The aim of research presented here was to assess the luminescence ability of benzanthrone derivatives using microscopic visualization of biological objects. Accordingly, specimens of freshwater trematodes: Diplostomum spathaceum, Diplodiscus subclavatus and Prosotocus confusus, were stained by novel benzanthrone dyes using different fixatives. The samples were examined under a confocal laser scanning microscope. All of the dyes tested demonstrated good results for digestive and reproductive system visualization. Based on obtained results we conclude that benzanthrone dyes could be used for internal and external structure confocal laser scanning microscopic imaging of trematode specimens.
\end{abstract}

Key words: benzanthrone dyes; trematode; confocal laser scanning microscopy

Received: 14 February, 2018; revised: 27 June, 2018; accepted: 12 July, 2018; available on-line: 27 August, 2018

e-mail: jelena.kirilova@du.lv

*Preliminary report presented: Diagnostic of parasites using novel luminescent dyes and confocal laser scanning microscopy. 6th Central European Congress of Life Sciences. EUROBIOTECH, 11-14 September, 2017, Krakow, Poland

Abbreviations: CLSM, confocal laser scanning microscopy; mtc., metacercariae

\section{INTRODUCTION}

Luminescence imaging techniques are becoming a popular tool to investigate the structure and properties of biological objects. Laser-induced fluorescence is a sensitive approach which gives an opportunity to detect even a single-molecule under appropriate conditions (Wu \& Dovichi, 1989; Van der Berg, 2000). Fluorescent molecular dyes, making lipid structures visible through optical techniques, are one of the tools currently used to study cell membranes. Despite a large number of various fluorescent probes that were constructed for bio-imaging (Albani, 2007), the existing membrane probes cannot satisfy the rapidly growing demands of researchers. Benzanthrone dyes attract particular interest due to their useful spectral properties (Carlini et al., 1982; Krasovitsky \& Bolotin, 1988). Technological utilization of these com- pounds is continuously growing, however, their applicability as fluorescent probes in biological assays still remains scantily evaluated. Meanwhile, spectral characteristics of benzanthrone dyes satisfy all the requirements for an ideal fluorescent marker. Bright fluorescence, high extinction coefficient, photo-, thermo- and chemical stability, and reduced background signal make benzanthrone dyes particularly attractive as bio-imaging agents (Yang et al., 1999). Our previous study demonstrated high lipidassociating ability of a series of newly synthesized benzanthrone amino and amidino derivatives (Trusova et al., 2012; Zhytniakivska et al., 2014).

The aim of the study presented here was to estimate new benzanthrone derivates as luminescent dyes for microscopic visualization of freshwater trematodes. Parasites are important components of biodiversity (Poulin \& Morand, 2000) and are suitable model organisms for evaluation of our new dyes and protocol elaboration. Moreover, some trematode species cause different human cancer forms, for example Clonorchis sinensis and Opistorchis viverrini can induce human cholangiocarcinoma, as well as induce other hepatobiliary pathologies (Petney et al., 2013). The spectroscopic properties of novel synthesized compounds were reviewed in this study. Descriptions of synthesis and characterization of new benzanthrone derivates as luminescent dyes are provided.

\section{MATERIALS AND METHODS}

Chemicals. Fluorescent benzanthrone dyes AM1 (3-N(N',N'-dimethylbenzamidino)benzanthrone), AM2 (3-N(N',N'-dimethylacetamidino)benzanthrone), AM4 (3-N(N',N'-diethylacetamidino)benzanthrone), AM16 (3-N-[N'(4-methylphenyl)acetamidino]benzanthrone), and P8 (3-Npiperidinobenzanthrone) were synthesized from 3-aminobenzanthrone or 3-bromobenzanthrone according to procedures described earlier (Kirilova et al., 2008; Kirilova et al., 2009; Gonta et al., 2013). The progress of chemical reactions and purity of products were monitored by thin-layer chromatography (TLC) on silica gel plates, Silufol UV254, $15 \times 15,0.2 \mathrm{~mm}$, using the solvent system of benzene/acetonitrile (3:1) as eluent. Column chromatography on silica gel was carried out on the Merck Kieselgel (230-240 mesh) with benzene as eluent. Stock solutions of benzanthrones were prepared by dissolving the dyes in ethanol or chloroform. All other chemicals were of analytical grade (Aldrich Chemical Company) and used without further purification. 
Model organisms. Fish and amphibian organs were investigated by compression method (Khalil et al., 2014) under a stereomicroscope SMZ800 (Nikon, Japan), magnification 15-30×. Freshwater trematode species: Diplostomum spathaceum, Diplodiscus subclavatus and Prosotocus confusus were collected from eyes, as well as digestive tracts by opening abdominal cavity of their respective hosts (Justine et al., 2012). Larval (metacercariae) and adult forms (marita) were used.

Fixation and staining. Each trematode species was fixed in a different fixative: $D$. spathaceum metacercariae (mtc.) in 96\% ethanol, adult D. subclavatus in Carnoy's solution (6:3:1 - absolute ethanol: chloroform: glacial acetic acid) adult $P$. confusus in AFA solution (17:2:1 - 85\% ethanol: formalin: glacial acetic; $\mathrm{pH}=4.5)$ for 1 hour, washed and stored in $96 \%$ ethanol at $4^{\circ} \mathrm{C}$ until required. The study design was as follows: all of the prepared specimens were stained in five different benzanthrone dyes: AM1, AM2, AM4, AM16 and P8 (dissolved in ethanol, molar concentration $10^{-4} \mathrm{M}$ ). After $10 \mathrm{~min}-$ utes, the specimens were washed three times with $70 \%$ ethanol and were dehydrated in 70, 80 and 96\% ethanol in the ascending order. Every step lasted 5-10 minutes, depending on thickness of the parasites' body wall. Next, ethanol-xylene (1:1) solution was used to obtain appropriate transparency changes under stereomicroscope. For the thickest specimens (D. subclavatus and $P$. confusus) an additional step with $100 \%$ xylene was used. Specimens were mounted in the Canada balsam (Sigma-Aldrich, $60610)$ and then were covered with a square coverslip $(24 \times 24)$, dried and kept in the dark until examination.

Microscopy. Microscopic observation was performed by using a high speed multiphoton confocal laser scanning microscope Nikon Eclipse Ti-E configured with A1 R MP inverted microscope system and equipped with digital sight DS-U3 camera (Nikon, Japan). Images of the specimens were processed using NIS Elements Advanced Research 3.2 64-bit software (Nikon, Japan). Slides were observed at various magnifications, from $\times 100$ to $\times 400$. Fluorescence was induced by using the following excitation laser wavelengths: (i) $\lambda=488$ $\mathrm{nm}$ with the FITC filter, (ii) $\lambda=561 \mathrm{~nm}$ with the TRITC filter. Objectives used: Plan-Apo 10×/0.45; Plan-Apo $20 \times / 0.75$; Plan-Apo 40×/0.95. Appropriate image modifications were obtained using Corel PHOTO-PAINT X6 (Corel Corporation, Canada).

\section{RESULTS}

The target dyes were synthesized in high yields by condensation of 3-amino-benzanthrone or 3-bromobenzanthrone with appropriate amidino or amino derivates. Chemical structures of the studied benzanthrone dyes are presented in Fig. 1.

The investigated substances belong to the class of solvatochromic dyes, which are among the oldest and most established membrane probes. These dyes exhibit strong changes in their dipole moments upon electronic excitation. Dipole-dipole interactions and specific interactions of these dye molecules with their environment change the energy of electronic transitions, and thus shift the maxima of their excitation and emission spectra (Kirilova et al., 2009; Gonta et al., Kirilova et al., 2008).

All of the five investigated benzanthrone dyes are suitable for confocal laser scanning microscopy (CLSM) of animal origin specimens. Not all combinations of dyes and fixatives were provided with pictures because of similar imaging results. Obviously, the target dyes as

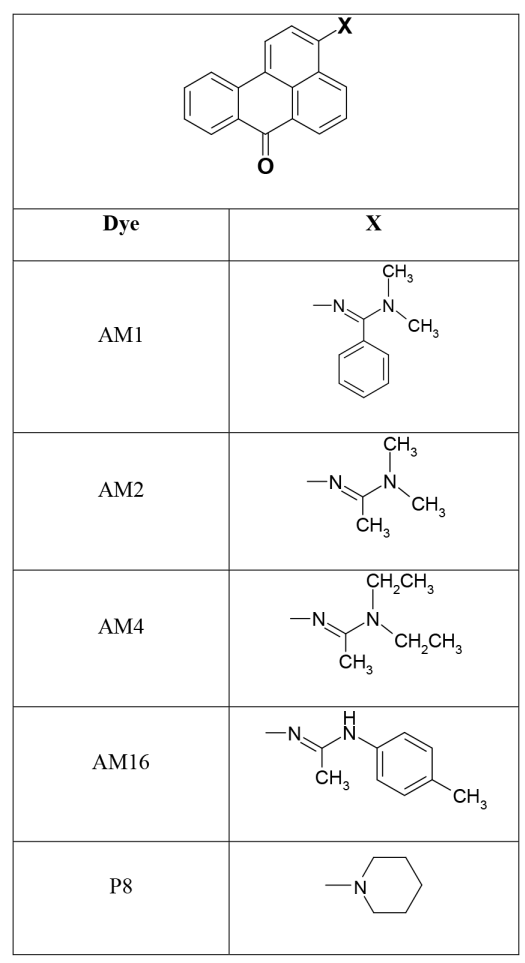

Figure 1. Chemical structures of the dyes used

highly lipophilic substances interacted with biological objects in the first association with the most hydrophobic parts of the cell - their membranes.

Microscopic examination of $D$. spathaceum mtc. fixed in $96 \%$ ethanol indicated high applicability of all studied luminescence dyes for visualization of the trematode species. The parasites' bodies were elongated and ovalshaped. Digestive tract stained very well in most parts of the mtc. specimens. Ventral sucker situated mid-ventrally. Under the ventral sucker, a relatively large holdfast organ was visualized. The oral sucker was located at anterior end of the body and continued into the muscular pharynx. Two intestinal caeca reached the end of the holdfast organ (Fig. 2). Two well developed pseudo suckers were visualized at each side of the oral suckers. The primary excretory system was stained (Figs. 2 and $3)$. In some of the mtc. Specimens, large amounts of rounded calcareous bodies were revealed which extended from the oral sucker to the holdfast organ (Fig. 3).

All stained specimens of adult $D$. subclavatus trematode in tandem with Carnoy's fixative, on the one hand, exhibited better results for visualization of small, taxonomically important skeletal elements, as well as oral and accessory suckers and cavity of the accessory sucker. On the other hand, these specimens did not show internal organs and structures at appropriate resolution level.

Target benzanthrone dyes, together with AFA fixative, displayed excellent visualization of internal and external structures. All of the revealed internal and external structures were clearly visible and easily recognizable. Change of focus gave an opportunity to get detailed images of different organs of $P$. confusus. External structure images showed that the parasites' bodies were round-shaped, cuticula was covered with spines (Fig. 4). Two of the same size suckers were revealed - an oral sucker, located at the anterior end of the body, and a ventral sucker situated mid-ventrally. Shape of cirrus (male copulatory organ) was clearly visible. 


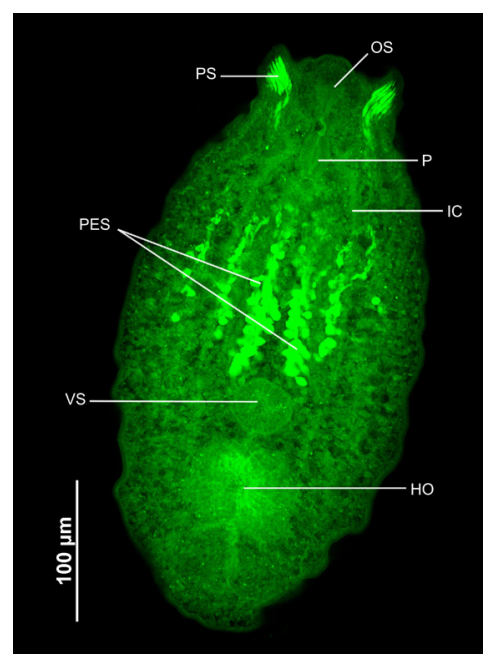

Figure 2. Diplostomum spathaceum mtc. stained with AM16 OS, oral sucker; PS, pseudo suckers; P, pharynx; IC, intestinal caeca; VS, ventral sucker; HO, holdfast organ; PES, primary excretory system. Picture obtained with $488 \mathrm{~nm}$ laser. Magnification $\times 200$; laser power 29.3; pinhole 54.1

Visualization of internal structure revealed that all of the variations of evaluated dyes and AFA fixative in adult $P$. confusus trematode specimens presented a well stained digestive system (Fig. 5). Directly behind the oral sucker, the pharynx bulbar was visualized which connected to the oesophagus, hidden under the eggs. It was bifurcated, forming two intestinal caeca. The intestines were shortened and did not reach the ventral sucker. Reproductive system also stained well in the adult parasite stage specimens. Cirrus, bursa and plenty of eggs were clearly visible throughout the length of the parasite. Reproductive bursa was large, located near the ventral sucker. The eggs were especially brightly fluorescent. Spines were also easily detected around the parasites' bodies.

\section{DISCUSSION}

Benzanthrone derivatives are polyaromatic hydrophobic substances. Due to planar structure and high lipophilicity of their molecules, they effectively interact with

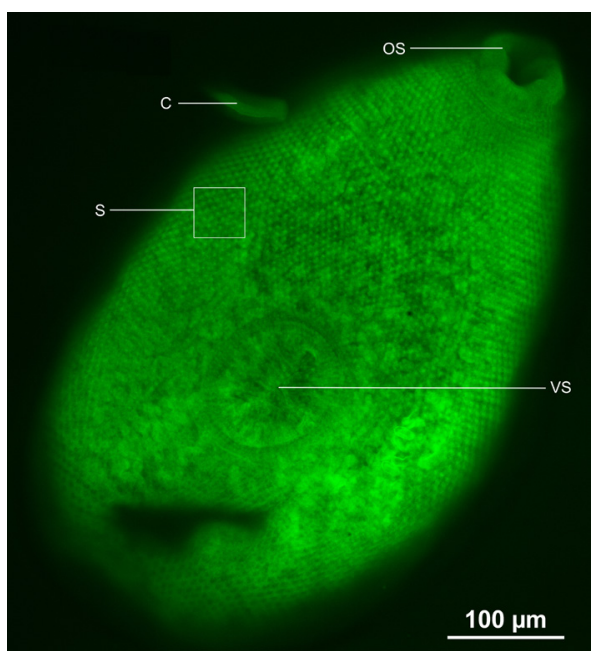

Figure 4. Adult Prosotocus confusus stained with AM1 (external structure)

S, spines; OS, oral sucker; VS, ventral sucker; $C$, cirrus. Picture was obtained with $488 \mathrm{~nm}$ laser. Magnification $\times 200$; laser power 36.2 ; pinhole 137.3

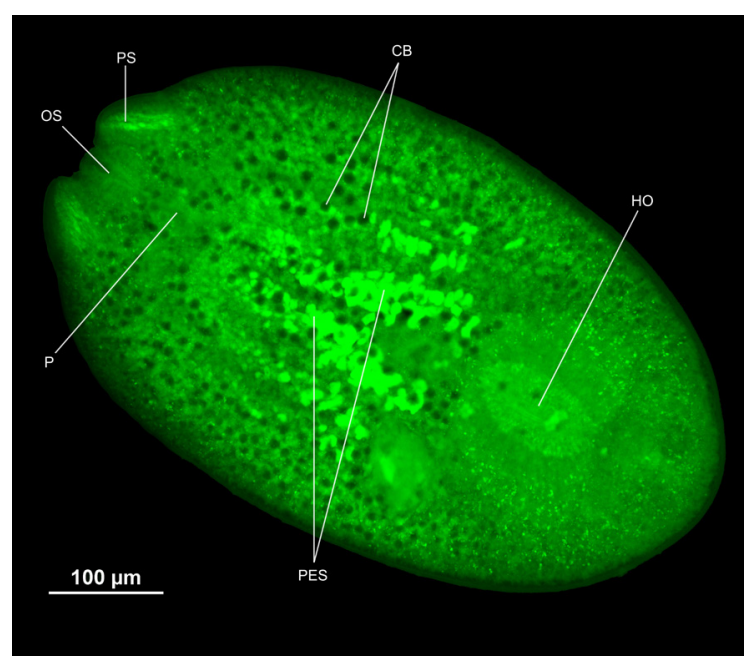

Figure 3. Diplostomum spathaceum mtc. stained with AM2 OS, oral sucker; PS, pseudo suckers; $\mathrm{P}$, pharynx; HO, holdfast organ; CB, calcareous bodies; PES, primary excretory system. Picture was obtained with $488 \mathrm{~nm}$ laser. Magnification $\times 200$; laser power 38.9; pinhole 158.4

membrane lipids. Nowadays, some benzanthrone derivatives are widely used as fluorescent dyes and probes. In this context, we decided to design new benzanthrone luminescent dyes to visualize cell membranes due to these dyes' localization within membrane lipids. Benzanthrone derivatives have many favorable photophysical properties, such as a significant Stokes shift, large extinction coefficient, bright fluorescence, low fluorescence intensity in buffer and high sensitivity of luminescence to the properties of the local environment (Kirilova et al., 2008, 2009; Gonta et al., 2013; Vus et al., 2014).

In previous studies absorption and emission spectra of target dyes were recorded in two different organic solvents and in solid state upon excitation of the samples at the lowest energy absorption band (Table 1). The electronic absorption spectra of the novel synthesized dyes showed bands around 250-280 $\mathrm{nm}$ and a broad long-wave band around 450-470 nm (log $e=3.80-4.60)$, which has a charge transfer character, due to $\pi \rightarrow \pi$ ' electron transfer during the $\mathrm{S}_{0} \rightarrow \mathrm{S}_{1}$ transition. The charge

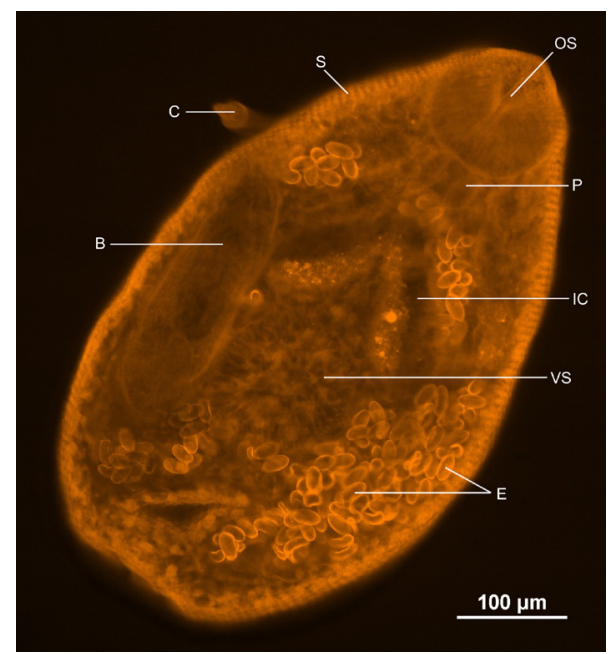

Figura 5. Adult Prosotocus confusus stained with AM1 (internal structure)

OS, oral sucker; $P$, pharynx; IC, intestinal caeca; VS, ventral sucker; E, eggs; S, spines; $B$, bursa; $C$, cirrus. Picture was obtained with 563 $\mathrm{nm}$ laser. Magnification $\times 200$; laser power 32.4 ; pinhole 127.6 
Table 1. Absorption and fluorescence data of the studied dyes at $10^{-5} \mathrm{M}$ in solutions

\begin{tabular}{|c|c|c|c|c|c|}
\hline \multirow{2}{*}{ Compound } & \multicolumn{2}{|c|}{ Absorption $\mathrm{I}_{\mathrm{abs}}(\log \mathrm{e}), \mathrm{nm}$} & \multicolumn{2}{|c|}{ Fluorescence $\mathrm{I}_{\mathrm{em}}, \mathrm{nm}$} & \multirow[t]{2}{*}{ References } \\
\hline & $\mathrm{CHCl}_{3}$ & $\mathrm{EtOH}$ & $\mathrm{CHCl}_{3}$ & $\mathrm{EtOH}$ & \\
\hline AM1 & $470.2(4.36)$ & $469.5(4.61)$ & 611 & 655 & \multirow{5}{*}{$\begin{array}{c}\text { Kirilova et al., 2009; Gonta et al., } \\
\text { Kirilova et al., } 2008\end{array}$} \\
\hline AM2 & $464.0(4.09)$ & $461.6(4.08)$ & 614 & 677 & \\
\hline AM4 & $467.0(4.17)$ & $472.8(4.14)$ & 615 & 670 & \\
\hline AM16 & $448(3.94)$ & $464(3.80)$ & 603 & 662 & \\
\hline P8 & $458(4.13)$ & $457(4.01)$ & 630 & 670 & \\
\hline
\end{tabular}

transfer in benzanthrone dyes occurs from the electron donor-acceptor interaction between electron-donating substituents at a C-3 position and the electron-accepting carbonyl group of the chromophoric system (Khrolova et al., 1984).

The compounds used are strongly fluorescent in solutions in the region of $603-630 \mathrm{~nm}$ (in chloroform) to 655-677 $\mathrm{nm}$ (in ethanol). The effect of the polarity of the medium on the fluorescence is more pronounced than on the absorption spectrum. This is because the intramolecular charge transfer effect leads to a large dipole moment in the excited state. The different behaviour in absorption and emission is related to the magnitude of the solvent effect on the energy of the ground and excised states during electron transition.

Later, several benzanthrone dyes were synthesized to verify changes in the physicochemical properties of a lipid bilayer (Trusova et al., 2012; Ryzhova et al., 2016), and to identify and characterize fibrillar aggregates of lysozyme (Vus et al., 2014).

At present, CLSM is widely used to study various species' morphological and physiological structures in fixed trematode specimens (Jurberg et al., 2008; Borges et al., 2017). Souza and coworkers (Souza et al., 2011) utilized hydrochloric carmine to observe trematode reproductive system by CLSM. Souza and coworkers (Souza et al., 2013) used CLSM to characterize morphology of encysted, activated, breached and excysted stages of metacercaria.

Nowadays, CLSM is an important method in biology studies and becomes more applicable in medicine investigations, e.g. cancer diagnostics, such as pancreatic cancer (Durko \& Małecka-Panas, 2015) and skin melanoma (Bragaa et al., 2012; Sqouros et al., 2014; Farnitani et al., 2015). Kirilova and coworkers (Kirilova et al., 2012) used benzanthrone dye ABM (analogue of dye P8) as fluorescent biomarker in colorectal cancer detection. The obtained results revealed that measurements of ABM spectral characteristics could be a potentially useful approach to estimate the immune status of gastrointestinal patients.

Our study showed no evidence of detailed visualization of body muscle structure, excluding muscular organ pharynx, and oral and ventral suckers. The general pattern of the trematode body wall musculature organization includes three muscle layers as follows: circular, longitudinal, and diagonal (Halton, 2014). More complicated methods are needed to detect muscle layers, e.g. Krupenko (2014) used 4\% solution of paraformaldehyde in phosphate-buffered saline (PBS) as fixative following by staining with TRITC-conjugated phalloidin for D. subclavatus specimens. Phalloidin has been found to bind to polymeric and oligomeric forms of actin. (Oda et al., 2005). Benzanthrone dyes are able to bind to such proteins as human serum albumin and amyloid fibrils of lysozyme as well (Gorbenko et al., 2010; Ryzhova et al., 2016).
Rozario and Newmark (Rozario \& Newmark, 2015) study demonstrated that the nervous system and other sensory structures of Hymenolepis diminuta tapeworm were stained with anti-synapsin antibodies. Like other flatworms, adult $H$. diminuta possesses a nervous system with both, central and peripheral components. Two lateral nerve cords, two median nerves and cephalic ganglia were well stained. In the study present here none of the nervous system parts were detected using each of the target dyes: AM1, AM2, AM4, AM16 and P8.

The obtained results demonstrate that the dehydratation step is crucially important, because presence of unsubstituted water in the cell structure prevents obtaining subsequent appropriate transparency. Shigin (Shigin, 1996) used a dehydratation step in staining trematodes for light microscopy, and the study presented here shows that dehydratation is applicable in newly developed protocol for CLSM.

Our results also revealed that parasites with thinwalled structures (e.g. Diplostomatidae family) do not require an additional step of enlightenment with 100\% xylene, since the absolute xylene can deform the shape of sensitive specimens. On the other hand, it is an optional step for parasites with thick wall, especially with external spines, which improves visualization of organs and inner structures.

It is required to use lasers in descending order of wave length to obtain better results.

With growing intensity of laser, structures become better visualized; however maximal intensity of lasers makes specimens absolutely unusable. Laser beam with a wavelength of $405 \mathrm{~nm}$ was not used in this study because its impact on biological objects is destructive and generates tissue auto fluorescence.

In previous investigations location of benzanthrone dyes in model membranes was revealed by Förster resonance energy transfer and red edge excitation shift approach (Zhitniakivska et al., 2014; Ryzhova et al., 2016). The obtained results show that due to high lipophilicity dye P8 can penetrate into hydrophobic region of the membrane. Dye P8 is situated in the phospholipid headgroup region, although other dyes prefer binding sites which are closer to the membrane surface. It was found by Ryzhova and coworkers (Ryzhova et al., 2016) that dyes AM2 and AM4 possess a considerably lower lipidassociating ability when compared to P8.

We have observed a small offset of maximal luminescence peak. It is important to keep in mind, that each cell of organism has autofluorescence in the blue-yellow region of spectrum and there may be interference with such fluorophores as proteins containing aromatic aminoacids, NAD $(\mathrm{P}) \mathrm{H}$, flavins and lipopigments (Monici, 2005). Our dyes experimentally demonstrated fluorescence in the red region of the spectrum, however, stained specimens had the displacement of fluorescence into a more short-wave region. That could be a result of more hydrophobic conditions (a higher content of lipids, 
dehydration by ethanol). Also, the action of the fixative may result in the appearance of side luminescence. For example, using formalin-containing fixative mixtures resulted in bright luminescence of cells in the yellow-green region of the spectrum (Alfano et al., 1984).

The investigated fluorophores were successfully applied to the fluorescent imaging of objects and identification of studied parasite species. These dyes present opportunities for biology researchers for further studies. In further studies we are planning to use more luminescent dyes for microscopic visualization of trematodes and optimize staining protocols.

\section{CONCLUSION}

In conclusion, the study presented here has been undertaken to evaluate the potential of the novel benzanthrone dyes to visualise trematode species. The target dyes absorb at 450-470 nm and emit at 603-677 nm. The target dyes displayed a positive solvatochromism and demonstrated their potential as luminescent probes for hydrophobic parts of the cell - membranes. Our results suggest a high compatibility of the new dyes tested with different common used fixatives, such as AFA and ethanol. These combinations gave good results of digestive and reproductive system imaging. Our study highlights the universalism and good staining properties of the benzanthrone dyes. They allow to get a clear visualisation of parasites' internal and external structures.

\section{Conflict of interest}

The authors declare no conflict of interest.

\section{Acknowledgements}

This work was supported by European Regional Development Fund (ERDF), Measure 1.1.1.1 "IndustryDriven Research”, Project No. 1.1.1.1/16/A/211 „Molecular design of new luminescent compounds for diagnostic purposes".

\section{REFERENCES}

Albani JR (2007) Principles and applications of fluorescence spectroscopy. Oxford, Blackwell. https://doi.org/10.1002/9780470692059

Alfano RR, Tata DB, Corsero J, Tomashefsky P, Longo FW, Alfano MA (1984) Laser induced fluorescence spectroscopy from native cancerous and normal tissue. IEEE J Quantum Elect 20: 1507-1511. https://doi.org/10.1109/JQE.1984.1072322

Borges N, Costa VS, Mantovani C, Barros E, Santos EGN, Marfa CL, Santos CP (2017) Molecular characterization and confocal laser scanning microscopic study of Pygidiopsis macrostomum (Trematoda: Heterophyidae) parasites of guppies Poecilia vivipara. J Fish Dis 40: 191-203. https://doi.org/10.1111/jfd.12504

Braga JC, Cao T, Oliviero MC, Duprata J, Rabinovitz HS, Rezze GG (2012) In vivo confocal microscopy: a promising diagnostic method for cutaneous oncology. J Clin Exp Dermatol Res S3. https://doi. org/10.4172/2155-9554.S3-002

Carlini F, Paffoni C, Boffa G (1982) New daylight fluorescent pigments. Dyes Pigm 3: 59-69. https://doi.org/10.1016/01437208(82)80013-2

Durko L, Małecka-Panas E (2015) The role of confocal microscopy in the diagnosis of pancreatic neoplasms. Postępy Nauk Medycznych 28: 38-41 (in Polish?)

Farnetani F, Scope A, Braun RP, Gonzalez S, Guitera P, Malvehy J, Manfredini M, Marghoob AA, Moscarella E, Oliviero M, Puig S, Rabinovitz HS, Stanganelli I, Longo C, Malagoli C, Vinceti M, Pellacani $G$ (2015) Skin cancer diagnosis with reflectance confocal microscopy reproducibility of feature recognition and accuracy of diagnosis. JAMA Dermatol 151: 1075-1080. https://doi.org/10.1001/ jamadermatol.2015.0810

Gonta S, Utinans M, Kirilov G, Belyakov S, Ivanova I, Fleisher M, Savenkov V, Kirilova E (2013) Fluorescent substituted amidines of benzanthrone: synthesis, spectroscopy and quantum chemi- cal calculations. Spectrochim Acta A 101: 325-334. http://dx.doi. org/10.1016/j.saa.2012.09.104

Gorbenko G, Trusova V, Kirilova E, Kirilov G, Kalnina I, Vasilev A, Kaloyanova S, Deligeorgiev T (2010) New fluorescent probes for detection and characterization of amyloid fibrils. Chem Phys Lett 495: 275-279. https://doi.org/10.1016/j.cplett.2010.07.005

Halton DW, Maule AG (2004) Flatworm nerve-muscle: structural and functional analysis. Can J Zool 82: 316-333. https://doi. org/10.1139/z03-221

Jurberg AD, Pascarelli BM, Pelajo-Machado M, Maldonado A Jr, Mota EM, Lenzi HL (2008) Trematode embryology: a new method for whole-egg analysis by confocal microscopy. Dev Genes Evol 218: 267-271. https://doi.org/10.1007/s00427-008-0209-0

Justine JL, Briand MJ, Bray RA (2012) A quick and simple method, usable in the field, for collecting parasites in suitable condition for both morphological and molecular studies. Parasitol Res 111: 341351. https://doi.org/10.1007/s00436-012-2845-6

Khalil MI, El-Shahawy IS, Abdelkader HS (2014) Studies on some fish parasites of public health importance in the southern area of Saudi Arabia. Rev Bras Vet Parasitol 23: 435-442. http://dx.doi. org/10.1590/S1984-29612014082

Khrolova OR, Kunavin NI, Komlev IV, Tavrizova MA (1984) Spectral and luminescence properties of phosphorylmethyl derivatives of 3-aminobenzathrone. J Appl Spectr 41: 771-775. https://doi. org/10.1007/BF00657690

Kirilova E, Kalnina I, Kirilov G, Gorbenko G (2012) Fluorescent biomarker in colorectal cancer. In Colorecatal Cancer Biology - from Genesis to Tumor. Ettarh R. eds, pp 429-446. China, InTech. https://doi. org $/ 10.5772 / 28733$

Kirilova EM, Belyakov SV, Kalnina I (2009) Synthesis and study of N,N-substituted 3-amidinobenzanthrones. In Topics in Chemistry \& Materials Science. Vayssilov G, Nikolova R eds, pp 19-28. Heron Press.

Kirilova EM, Kalnina I, Kirilov GK, Meirovics I (2008) Spectroscopic study of benzanthrone 3-N-derivatives as new hydrophobic fluorescent probes for biomolecules. J Fluoresc 18: 645-648. https://doi. org/10.1007/s10895-008-0340-3

Krasovitsky BM, Bolotin BM (1988) Organic luminescent materials. NY, VCH Publishers

Krupenko DY (2014) Muscle system of Diplodiscus subclavatus (Trematoda: Paramphistomida) cercariae, pre-ovigerous, and ovigerous adults. Parasitol Res 113: 941-952. https://doi.org/10.1007/s00436013-3726-3

Monica M (2005) Cell and tissue autofluorescence research and diagnostic applications. Biotechnol Annu Rev 11: 1387-2656. https://doi. org/10.1016/S1387-2656(05)11007-2

Oda T, Namba, Maéda Y (2005) Position and orientation of phalloidin in $\mathrm{F}$-actin determined by x-ray fiber diffraction analysis. Biophys $J \mathbf{8 8}$ : 2727-2736. http://dx.doi.org/10.1529/biophysj.104.047753

Petney TN, Andrews RH, Saijuntha W, Wenz-Mücke A, Sithithaworn P (2013) The zoonotic, fish-borne liver flukes Clonorchis sinensis, Opisthorchis felineus and Opisthorchis viverrini. Int J Parasitol 43: 10311046. http://dx.doi.org/10.1016/j.ijpara.2013.07.007

Poulin R, Morand S (2000) The diversity of parasites. Q Rev Biol 75: 277-293. https://doi.org/10.1086/393500

Rozario T, Newmark PA (2015) A confocal microscopy-based atlas of tissue architecture in the tapeworm Hymenolepis diminuta. Exp Parasitol 158: 31-41. https://doi.org/10.1016/j.exppara.2015.05.015

Ryzhova O, Vus K, Trusova V, Kirilova E, Kirilov G, Gorbenko G, Kinnunen P (2016) Novel benzanthrone probes for membrane and protein studies. Methods Appl Fluoresc 4: 034007. https://doi. org/10.1088/2050-6120/4/3/034007

Sgouros D, Pellacani G, Katoulis A, Rigopoulos D, Longo C (2014) Confocal microscopy in diagnosis and management of melasma: review of literature. J Pigment Disord S1: 004. http://dx.doi. org/10.4172/JPD.S1-005

Shigin AA (1996) Morphological criteria of the species in cercaria of the genus Diplostomum (Trematoda: Diplostomidae) and methods for their study. Parazitologija 30: 425-439 (what language?).

Souza J, Garcia J, Neves RH, Machado-Silva JR, Maldonado A (2013) In vitro excystation of Echinostoma paraensei (Digenea: Echinostomatidae) metacercariae assessed by light microscopy, morphometry and confocal laser scanning microscopy. Exp Parasitol 135: 701-707. https://doi.org/10.1016/j.exppara.2013.10.009

Souza J, Garcia JS, Manso PP, Neves RH, Maldonado A Jr, MachadoSilva JR (2011) Development of the reproductive system of Echinostoma paraensei in Mesocricetus auratus analyzed by light and confocal scanning laser microscopy. Exp Parasitol 128: 341- 346. https://doi. org/10.1016/j.exppara.2011.04.005

Trusova V, Kirilova E, Kalnina I, Kirilov G, Zhytniakivska O, Fedorov P, Gorbenko G (2012) Novel benzanthrone aminoderivatives for membrane studies. J Fluoresc 22: 953-959. https://doi.org/10.1007/ s10895-011-1035-8

Van der Berg A, Olthuis W, Bergveld P (2000) Micro total analysis systems 2000. Dordrecht, Springer. https://doi.org/10.1007/978-94-0172264-3 
Vus K, Trusova V, Gorbenko G, Sood R, Kirilova E, Kirilov G, Kalnina I, Kinnunen P (2014) Fluorescence investigation of interactions between novel benzanthrone dyes and lysozyme amyloid fibrils. J Fluoresc 24: 493-504. https://doi.org/10.1007/s10895-013-1318-3

Wu S, Dovichi NJ (1989) High-sensitivity fluorescence detector for fluorescein isothiocyanate derivatives of amino acids separated by capillary zone electrophoresis. J Chromatogr A 480: 141-155. https:// doi.org/10.1016/S0021-9673(01)84284-9

Yang X, Liu W-H, Jin W-J, Shen G-L, Yu R-Q (1999) DNA binding studies of a solvatochromic fluorescence probe 3-methoxybenzanthrone. Spectrochim Acta A 55: 2719-2727. https://doi.org/10.1016/ S1386-1425(99)00161-4
Zhytniakivska O, Trusova V, Gorbenko G, Kirilova E, Kalnina I, Kirilov G, Molotkovsky J, Tulkki J, Kinnunen P (2014) Location of novel benzanthrone dyes in model membranes as revealed by resonance energy transfer. J Fluoresc 24: 899-907. https://doi. org/10.1007/s10895-014-1370-7

Zhytniakivska O, Trusova V, Gorbenko G, Kirilova E, Kirilov G, Kalnina I, Kinnunen P (2014) Newly synthesized benzanthrone derivatives as prospective fluorescent membrane probes. I Luminesc 146: 307-313. https://doi.org/10.1016/j.jlumin.2013.10.015 